\title{
The BaBar Detector at the SLAC B-Factory
}

\author{
D.H. Fujino
}

This paper was prepared for submittal to the

Sixth Conference on the Intersection of Particle and Nuclear Physics Big Sky, MT

May 22-June 2, 1997

July 1997

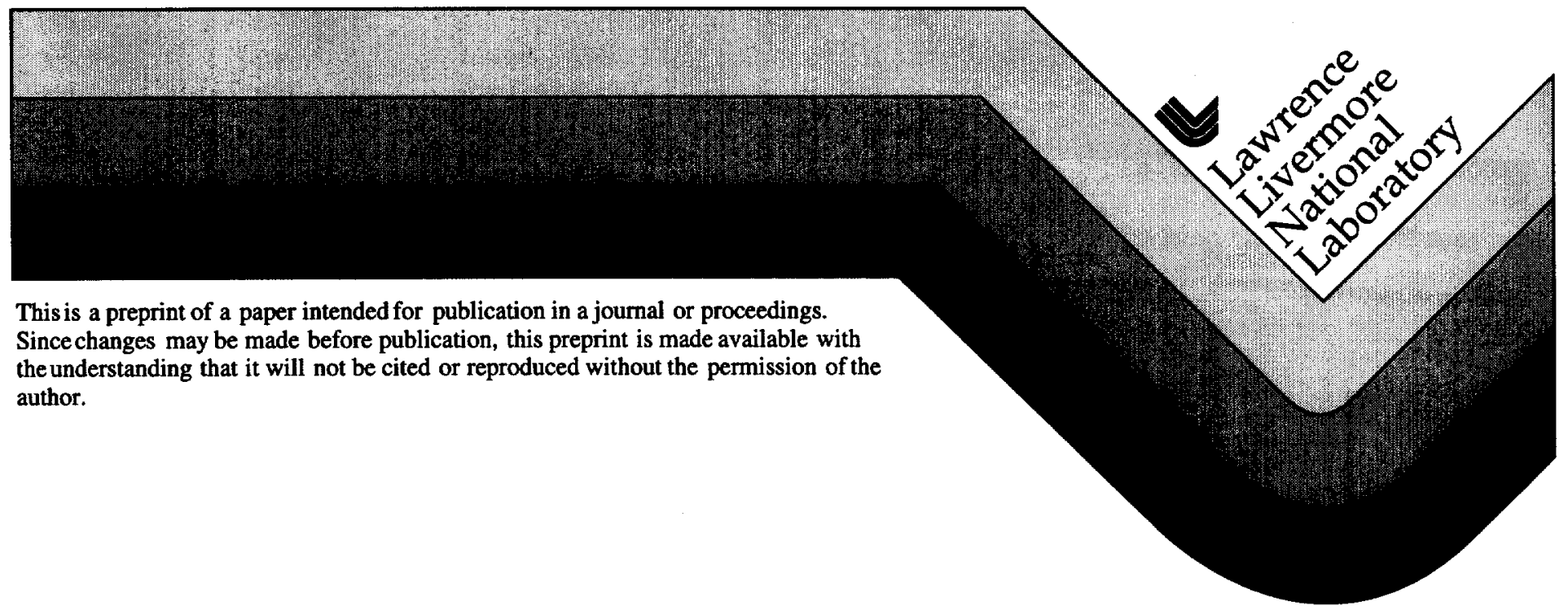




\section{DISCLAIMER}

This document was prepared as an account of work sponsored by an agency of the United States Government. Neither the United States Government nor the University of California nor any of their employees, makes any warranty, express or implied, or assumes any legal liability or responsibility for the accuracy, completeness, or usefulness of any information, apparatus, product, or process

disclosed, or represents that its use would not infringe privately owned rights. Reference herein to any specific commercial product, process, or service by trade name, trademark, manufacturer, or otherwise, does not necessarily constitute or imply its endorsement, recommendation, or favoring by the United States Government or the University of California. The views and opinions of authors expressed herein do not necessarily state or reflect those of the United States Government or the University of California, and shall not be used for advertising or product endorsement purposes. 


\title{
The BaBar Detector at the SLAC B-Factory
}

\author{
Don H. Fujino ${ }^{1}$ \\ Lawrence Livermore National Laboratory \\ Livermore, $C A 94550$ \\ Representing the BaBar Collaboration
}

\begin{abstract}
The SLAC B-Factory will search for CP violation in $B$ decays. I will describe the $\mathrm{BaBar}$ Detector and its current status.
\end{abstract}

\section{INTRODUCTION}

One of the Holy Grails in particle physics is to understand the nature of $\mathrm{CP}$ violation. CP violation can arise naturally in the Standard Model from a complex phase in the Cabibbo-Kobayashi-Maskawa (CKM) matrix, which describes the weak couplings of the six quarks. The BaBar experiment ${ }^{2}$ at the SLAC Asymmetric B-Factory is designed to observe CP violation in $B$ meson decays that arise from $\Upsilon(4 S) \rightarrow B^{0} \bar{B}^{0}$.

When a neutral $B$ meson decays into a $\mathrm{CP}$ eigenstate such as $B^{0} \rightarrow \psi K_{S}^{0}$, $\psi K_{L}^{0}$, or $\pi^{+} \pi^{-}$, it can decay directly into that final state, or it can mix into a $\bar{B}^{0}$ before decaying: $B^{0} \rightarrow \bar{B}^{0} \rightarrow \psi K_{S}^{0}$. Through interference effects from $B^{0}-$ $\bar{B}^{0}$ mixing, $\mathrm{CP}$ violation will introduce a time-dependent decay asymmetry between $B^{0} \rightarrow f_{C P}$ and $\bar{B}^{0} \rightarrow f_{C P}$, where $f_{C P}$ is a CP eigenstate. The usual exponential decay behavior becomes modified by subtle oscillations in the decay lifetime differences.

$$
\begin{aligned}
& \Gamma\left(B^{0} \bar{B}^{0} \rightarrow B^{0} f_{C P}\right) \propto \mathrm{e}^{-\Gamma \Delta t}[1+\sin \phi \sin (\Delta M \Delta t)] \\
& \Gamma\left(B^{0} \bar{B}^{0} \rightarrow \bar{B}^{0} f_{C P}\right) \propto e^{-\Gamma \Delta t}[1-\sin \phi \sin (\Delta M \Delta t)]
\end{aligned}
$$

where $\sin \phi$ is the CP violating parameter, $\Delta t$ is the decay time difference between the two $B$ mesons from $\Upsilon(4 S) \rightarrow B^{0} \bar{B}^{0}$ decays, and $\sin (\Delta M \Delta t)$ is the decay time oscillation from mixing.

1) This work performed under the auspices of the US Department of Energy by the Lawrence Livermore National Laboratory under Contract W-7405ENG-48.

2) For further details see the BaBar Technical Design Report, SLAC-R-95-457. 


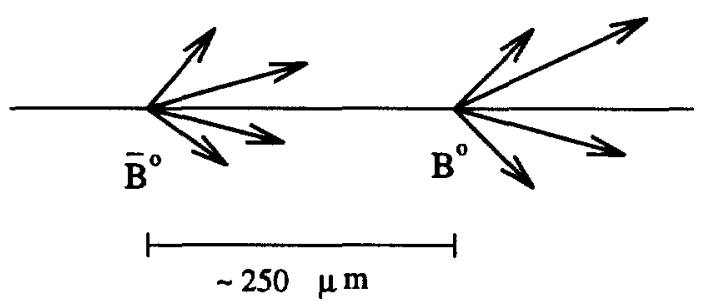

FIGURE 1. Vertex separation for $\Upsilon(4 S) \rightarrow B^{0} \bar{B}^{0}$ decays in an asymmetric collider.

In the center-of-mass frame of the $\Upsilon(4 S)$, the $B$ 's are nearly at rest, so there is no information on $B$ decay times and the $\mathrm{CP}$ violating effects are lost. However, SLAC will boost the $\Upsilon(4 S)$ frame by colliding a $9.0 \mathrm{GeV}$ electron beam with a $3.1 \mathrm{GeV}$ positron beam, so that the two $B$ mesons will decay along the beam axis with an average separation of $\sim 250 \mu \mathrm{m}$ (Figure 1.) This separation is easily resolved with a silicon vertex detector.

The BaBar detector must therefore satisfy three requirements:

1. Reconstruct $B$ decays into CP eigenstates $\left(\psi K_{S}^{0}, \psi K_{L}^{0}, \pi^{+} \pi^{-}\right.$, etc.)

2. Tag the flavor of the other $B$ meson using charged kaons or leptons

3. Measure the relative decay time $\Delta t=t_{2}-t_{1}$ of the two $B$ mesons

\section{THE BaBar DETECTOR}

The SLAC B-Factory will achieve a peak luminosity of $3 \times 10^{33} \mathrm{~cm}^{-2} \mathrm{~s}^{-1}$ by colliding $9.0 \mathrm{GeV} e^{-}$and $3.1 \mathrm{GeV} e^{+}$beams. Such high luminosities and asymmetric beams require high currents ( $\left.2 \operatorname{amp} e^{-}, 1 \operatorname{amp} e^{+}\right), 1700$ bunches, 4 ns beam crossings, and separate high and low energy rings to transport the beams without introducing any parasitic crossing. A final bending magnet in the interaction region (IR) will ensure the $e^{+} e^{-}$beams collide head-on. The bending near the IR will generate intense synchrotron radiation that is not present in conventional $e^{+} e^{-}$machines.

The BaBar Collaboration is made up of 10 Countries, 78 Institutions and over 500 physicists. The BaBar detector has 6 major subsystems. Going out radially, $\mathrm{BaBar}$ has a silicon vertex tracker, a drift chamber, a Cerenkov detector for $\pi / K$ separation, a CsI electromagnetic calorimeter, a 1.8 Tesla superconducting magnetic coil, and an instrumented flux return to detect muons and $K_{L}^{0}$. The detector is shown in Figure 2.

The Silicon Vertex Tracker (SVT) is a 5 layer double-sided detector which provides both $\phi$ and $z$ information. It will locate each $B$ decay vertex with a $\Delta Z$ resolution of $80 \mu \mathrm{m}$, and so will easily be able to resolve the $250 \mu \mathrm{m}$ average separation between the two $B$ mesons. The five layers are at $3,4,5$, 12 , and $14 \mathrm{~cm}$ in radius; the outer two layers are arched to minimize the area of silicon required and to avoid large incident track angles. (See Figure 3). 


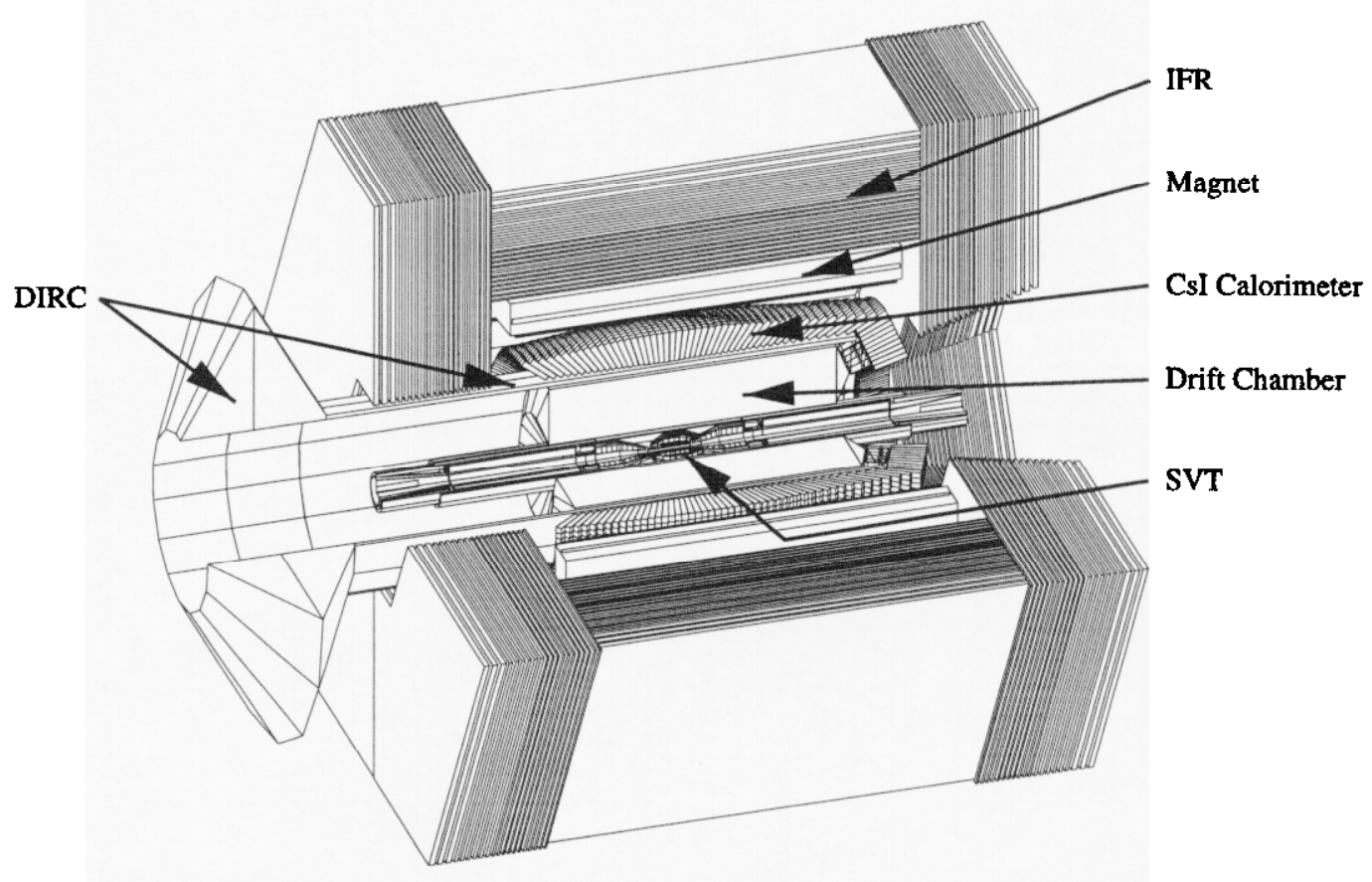

FIGURE 2. The BaBar detector.

The SVT will be able to find tracks independent of the drift chamber. It has $0.94 \mathrm{~m}^{2}$ of silicon strips and 150,000 readout channels. Because the SVT will be in a high synchrotron radiation background, $33 \mathrm{KRad} /$ year for the inner layer, the chips were designed rad-hard and AC coupled to reduce the noise. The SVT is constructed in two clam-shells which are mounted over the beampipe and the two conical bending magnets in the IR. A low mass space frame, constructed from carbon fiber rods spanning the length of the SVT, will provide the overall stiffness to the detector.

Just outside the SVT is the central drift chamber (DC). It must provide excellent momentum resolution for charged tracks in the range $100 \mathrm{MeV} / \mathrm{c}$ to $2.5 \mathrm{GeV} / \mathrm{c}$ to obtain good mass resolution for reconstructing exclusive $B$ decays. The $100 \mathrm{MeV} / \mathrm{c}$ lower limit is defined by the inner radius of the drift chamber; lower momentum tracks must rely primarily on the SVT. The DC momentum resolution is estimated to be $\sigma_{P_{T}} / P_{T}=0.21 \%+0.14 \% \times P_{T}$. The constant term is from multiple Coulomb scattering and is kept minimal since

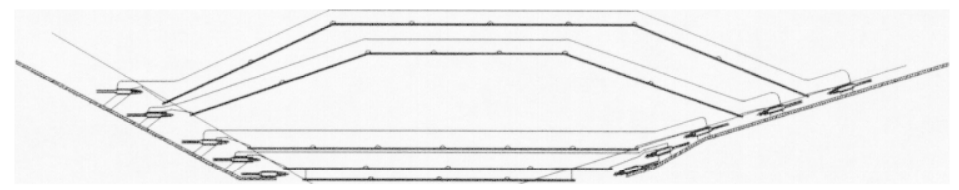

FIGURE 3. The 5-layer silicon vertex tracker. 


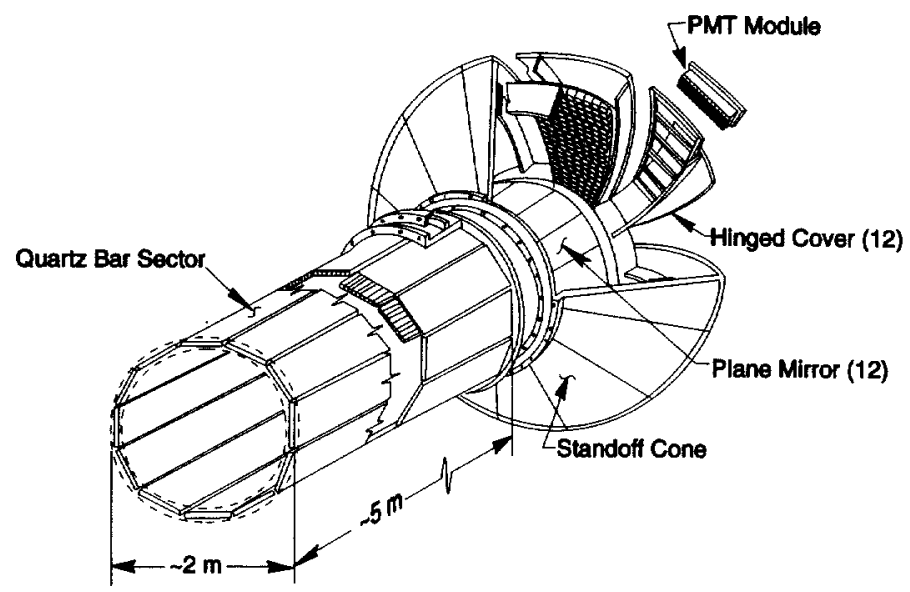

FIGURE 4. The DIRC detector.

most of the tracks from $B \bar{B}$ events have low momentum. This is achieved by using a helium-based gas in the chamber ( $80 \%$ helium, $20 \%$ isobutane), aluminum field wires, and an inner wall made of beryllium. The helium-based gas has a spatial resolution that is $\sim 30 \%$ better than convential DC gases such as HRS gas (Ar: $\left.\mathrm{CO}_{2}: \mathrm{CH}_{4}=89: 10: 1\right)$. The drift chamber uses small cells arranged in 40 layers over a radius of $23-80 \mathrm{~cm}$. They are grouped in 10 superlayers (AUVAUVAUVA), where $A$ is an axial superlayer and UV are stereo superlayer pairs with a stereo angle of $\pm 50 \mathrm{mrad}$.

Particle identification in the barrel region is provided by the Detector of Internally Reflected Cerenkov light (DIRC). The DIRC yields better than $4 \sigma$ separation for charged kaons and pions over the entire momentum and $\cos \theta$ range for $\Upsilon(4 S) \rightarrow B \bar{B}$ events. The drift chamber will provide limited $\pi / K$ separation from specific ionization $(d E / d x)$ information: over $3 \sigma$ separation for $P<0.7 \mathrm{GeV} / \mathrm{c}$ and about $2 \sigma$ separation for $P>3 \mathrm{GeV} / \mathrm{c}$; however, it offers no discrimination for intermediate momenta. Kaon identification is crucial for tagging the flavor of the unreconstructed $B$ meson and for distinguishing the CP eigenstate decay $B^{0} \rightarrow \pi^{+} \pi^{-}$from the penguin decay $B^{0} \rightarrow K^{+} \pi^{-}$.

The DIRC is situated in the $10 \mathrm{~cm}$ gap between the drift chamber and the calorimeter and uses a novel method for Cerenkov ring imaging. The DIRC consists of 156 long quartz bars $\left(1.75 \times 3.5 \times 470 \mathrm{~cm}^{3}\right)$ as Cerenkov radiators arranged in azimuth around the beamline (See Figure 4). Charged particles passing through the quartz bars radiate Cerenkov light which is internally reflected down the bar. The light exits in the backward region with its angular information still preserved into a large expansion region called the standoff cone. Six tons of water fill this region and the Cerenkov image expands $\sim 120$ $\mathrm{cm}$ to an array of 13400 photomultiplier tubes.

The CsI Calorimeter must reconstruct photons with high efficiency and energy resolution over a large dynamic range of $20 \mathrm{MeV}$ to $5 \mathrm{GeV}$. Most photons from generic $B$ decays are under $0.5 \mathrm{GeV}$, but those from $B^{0} \rightarrow \pi^{0} \pi^{0}$ 


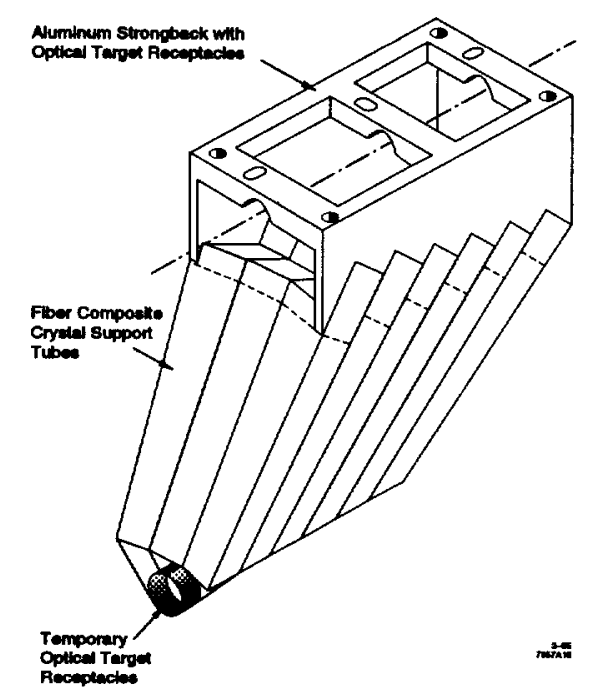

FIGURE 5. Calorimeter barrel module which houses an array of $3 \times 7 \mathrm{CsI}(\mathrm{Tl})$ crystals.

decays can have energies up to $4 \mathrm{GeV}$. The energy resolution is estimated to be $\sigma_{E} / E=1 \% / E(G e V)^{0.25} \oplus 1.2 \%$, about a factor of two better than the CLEO CsI calorimeter. In addition, the calorimeter must reconstruct $\pi^{0}$ 's and identify electrons with momenta as low as $P>0.5 \mathrm{GeV} / \mathrm{c}$. The calorimeter is composed of $5760 \mathrm{CsI}(\mathrm{Tl})$ crystals arranged for the barrel and 900 crystals for the forward endcap. The barrel covers the range $-0.80<\cos \theta_{l a b}<0.89$ and the forward endcap extends the coverage to $\cos \theta_{l a b}=0.97$. The barrel crystals are non-projective in $\cos \theta$ to reduce inefficiencies from gaps between crystals. The crystals are $16-17.5$ radiation lengths, longer in the forward region. Two photodiodes on the back face of the crystal measure the light output. An array of $3 \times 7$ crystals are housed in a carbon fiber module. (See Figure 5). An aluminum support is bonded to the back of each module and is rigidly attached to an outer cylindrical strongback structure.

The Instrumented Flux Return (IFR) provides muon and $K_{L}^{0}$ identification. Muons will be identified for $P>0.6 \mathrm{GeV} / \mathrm{c}$, significantly better than the limit of $P>1.4 \mathrm{GeV} / \mathrm{c}$ for unsegmented iron absorbers. $K_{L}^{0}$ mesons that interact in the IFR will have their direction measured from the energy deposition, but not their momentum. This is important for observing CP asymmetries in $B^{0} \rightarrow$ $\psi K_{L}^{0}$ decays. The IFR detector is composed of one barrel (in sextants) and two endcaps. There are a total of 20 layers of iron plates which increase in thickness from $2 \mathrm{~cm}$ in the inner layers to $5 \mathrm{~cm}$ in the outer layers. This layout optimizes the muon and $K_{L}^{0}$ finding efficiency. Resistive Plate Chambers (RPC's) were chosen to instrument the $2500 \mathrm{~m}^{2}$ of surface area since this technology is simple, reliable, and inexpensive (see Figure 6). RPC's have a $2 \mathrm{~mm}$ gas gap filled with Argon/Isobutane/Freon at atmospheric pressure between two layers of Bakelite with bulk resistivity of $\sim 10^{11} \Omega-\mathrm{cm}$. The two electrode plates are coated with a thin layer of graphite and connected to high voltage and to 


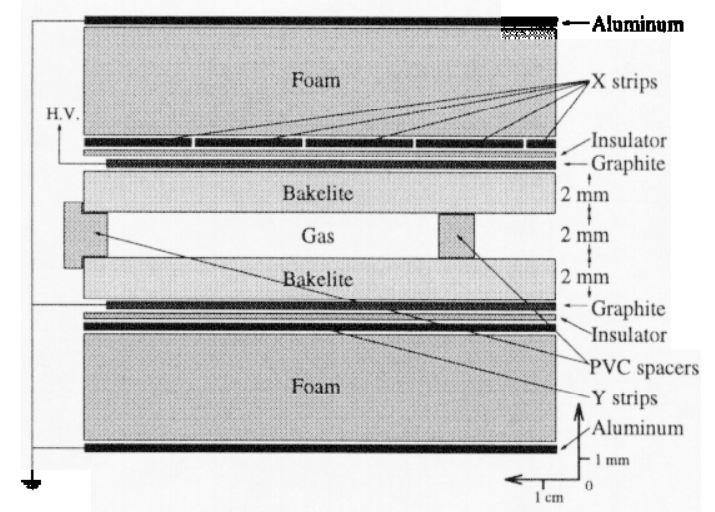

FIGURE 6. The RPC components in the IFR detector.

ground. Charged tracks generate a quenched spark that induces charge on $\mathrm{X}$ and Y pickup strips.

\section{CONCLUSIONS}

There is still a mountain of work to be accomplished before the B-Factory and $\mathrm{BaBar}$ detector are operational; however, the schedule has suffered no major slippages. The PEP II accelerator is ahead of schedule. The high energy ring has been built and is being commissioned. Magnets and vacuum systems are now being installed in the low energy ring. Progress continues towards completing the BaBar detector by the end of 1998 . The steel for the magnetic flux returns has arrived at SLAC and the RPC's are being assembled into place. Many of the major items such as the low mass space frame for the SVT, the drift chamber endplates and robotic wire stringers, and the calorimeter structural support cylinder are either built or being fabricated. The silicon wafers, DIRC quartz ingots, and CsI(Tl) crystals are in full production. With the BaBar detector scheduled to move into the beamline by 1999 , we will be in an excellent position to discover CP violation in $B$ decays.

Acknowledgements: I would like to thank C. Wuest and B. Wisniewski for useful and stimulating discussions. 


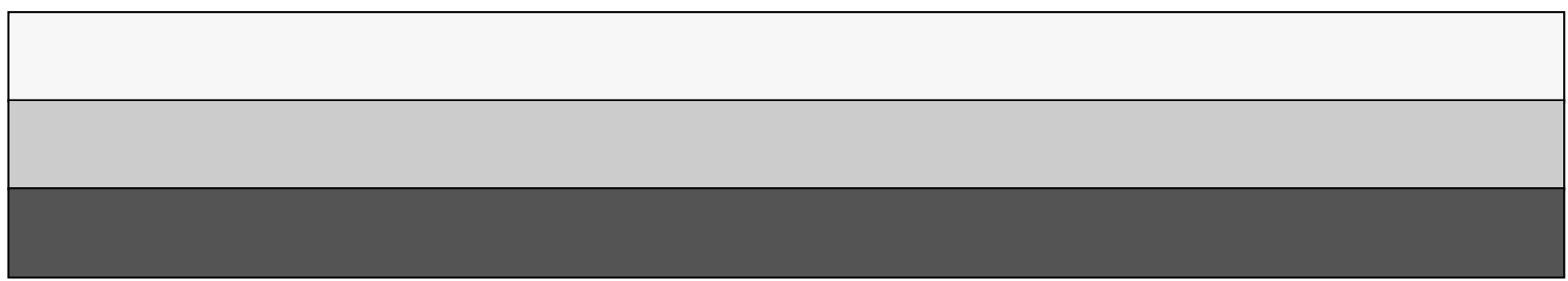

